\title{
RESEARCH HIGHLIGHT Dimethyl fumarate: targeting glycolysis to treat MS
}

\author{
Stefano Angiari ${ }^{1}$ and Luke A. O'Neill ${ }^{1}$ \\ Cell Research (2018) 28:613-615; https://doi.org/10.1038/s41422-018-0045-3
}

\begin{abstract}
Dimethyl fumarate (DMF) is an immunomodulatory drug used in patients with multiple sclerosis (MS) and psoriasis. Kornberg and colleagues shed new light on DMF activity, demonstrating that it blocks immune cell activation through inhibition of glycolysis via post-transcriptional modification of glyceraldehyde 3-phosphate dehydrogenase (GAPDH), which confirms the pro-inflammatory role of glycolysis and points to potential new targets to treat inflammatory diseases such as MS.
\end{abstract}

Dimethyl fumarate (DMF) is a fumaric acid ester used for decades for the treatment of psoriasis and recently approved as a disease-modifying drug in relapsing-remitting multiple sclerosis (MS), but its precise mechanisms of action is still a point of debate. ${ }^{1,2}$ The basis for DMF activity is further complicated by its hydrolysis in vivo to monomethyl fumarate (MMF), which can itself modulate immune responses and tissue inflammation., ${ }^{1,2}$ Many studies investigated the basis for DMF-induced immunomodulation, showing a plethora of effects on both tissue and immune cells. ${ }^{1,2}$ Possible targets include the engagement of antioxidant responses through activation of Nuclear factor (erythroid-derived 2)-like 2 (Nrf2), inhibition of nuclear factor kappa-light-chainenhancer of activated $B$ cells (NF-kB) and engagement with a putative receptor called HCA2., ${ }^{1,2}$ Of note, DMF treatment in both psoriasis and MS patients causes an immune switch characterized by induction of anti-inflammatory/regulatory innate and adaptive immune cell populations such as regulatory $T$ cells (Tregs) and inhibition or apoptosis of pro-inflammatory cells, such as T helper 1 (Th1) and Th17 subsets, ${ }^{1,2}$ highlighting its immunomodulatory properties.

Kornberg and co-workers now add a new possible target to the mix, demonstrating that DMF and its derivative MMF influence the intracellular metabolic state of activated innate and adaptive immune cells by inhibiting what might be considered an unlikely target, the enzyme glyceraldehyde 3-phosphate dehydrogenase (GAPDH), which is known as a loading control in western blots. The authors demonstrate that DMF and MMF covalently modify GAPDH in a reaction termed succination. ${ }^{3}$ Protein succination is a covalent post-transcriptional modification in which a molecule of fumarate, an intermediate metabolite of the tricarboxylic acid (TCA) cycle, reacts with a cysteine residue, forming a [S-(2-succino) cysteine] group. Many different proteins can be succinated by endogenous fumarate, and this reaction may significantly impact their functionality. ${ }^{4}$ Of note, GAPDH was previously reported to be succinated on the cysteine-152 in the active site, a modification that leads to the irreversible inactivation of its enzymatic activity, ${ }^{5}$ whereas DMF was shown to induce mouse tubulin succination in vitro. ${ }^{6}$ By using a mass spectrometry approach, Kornberg and colleagues confirmed that GAPDH is succinated by endogenous fumarate in both mice and human cells, and report that oral treatment with DMF or MMF further induced GAPDH succination in vivo in mice and in peripheral blood mononuclear cells from MS patients treated with DMF (Fig. 1). ${ }^{3}$ As expected, succination by DMF and MMF decreased GAPDH enzymatic activity in vitro and in vivo, confirming previous observations. $^{5}$

GAPDH catalyzes the conversion of glyceraldehyde 3-phosphate to 1,3-bisphosphoglycerate during glycolysis, an essential metabolic pathway where glucose is converted to pyruvate, to fuel the TCA cycle and oxidative phosphorylation (OXPHOS) for the production of adenosine triphosphate (ATP) as an energy source. Mammalian cells potentiate glycolysis in hypoxic conditions, when levels of free oxygen are low and cannot support an efficient OXPHOS. However, activated immune cells, as well as cancer cells, display high rates of glycolysis even in the presence of sufficient oxygen, a condition known as aerobic glycolysis. This metabolic switch, known as the Warburg effect, allows the cells to generate metabolic intermediates essential for their activation, proliferation and immune functions. ${ }^{7}$ Kornberg and co-workers showed that treatment with DMF or MMF blocks glycolysis at the level of GAPDH in both lipopolysaccharide (LPS)-activated peritoneal macrophages (PMs) and anti-CD3/anti-CD28-stimulated $T$ cells, with accumulation of pre-GAPDH metabolites and reduced levels of post-GAPDH ones, as demonstrated by $\mathrm{U}^{13} \mathrm{C}$-glucose tracing and metabolomics analysis (Fig. 1). ${ }^{3}$ This inhibition was confirmed via Seahorse experiments that showed less lactate production and lower glycolytic capacity in DMF- and MMF-treated cells, supporting recent observations showing reduced glycolysis in $T$ cells from MS patients treated with DMF.,8 Importantly, treatment of PMs with 2-deoxyglucose (a glycolysis inhibitor) or heptelidic acid (HA; a GAPDH inhibitor) mirrored the effect of DMF treatment, confirming that its immunomodulatory activity is likely to be due to an inhibition of glycolysis in activated immune cells. ${ }^{3}$ Of note, DMF did not alter the activation of two essential signaling drivers of glycolysis, mammalian target of rapamycin (mTOR) or hypoxia-inducible factor-1a (HIF-1a), further suggesting that its activity is due to inhibition of GAPDH. ${ }^{3,7}$ Interestingly, DMF, but not MMF, also increased OXPHOS rates in resting or activated PMs, an increase that was not the driver of the reduced glycolytic capacity. ${ }^{3}$ However, the authors did not investigate whether this increase in OXPHOS was a direct consequence of glycolysis blockade or was rather due to fumarate fueling into the TCA cycle by DMF.

Engagement of aerobic glycolysis by activating $T$ cells is an essential step for their proliferation and effector functions. ${ }^{7}$ However, different $T$ cell subsets present different reliance on aerobic glycolysis, with pro-inflammatory Th1 and Th17 cells being highly dependent on glycolysis and anti-inflammatory Tregs using alternative pathways such as fatty acid oxidation for their energy

${ }^{1}$ School of Biochemistry and Immunology, Trinity Biomedical Sciences Institute, Trinity College Dublin, Dublin 2, Ireland
Correspondence: Stefano Angiari (ANGIARIS@tcd.ie)

Published online: 29 May 2018 


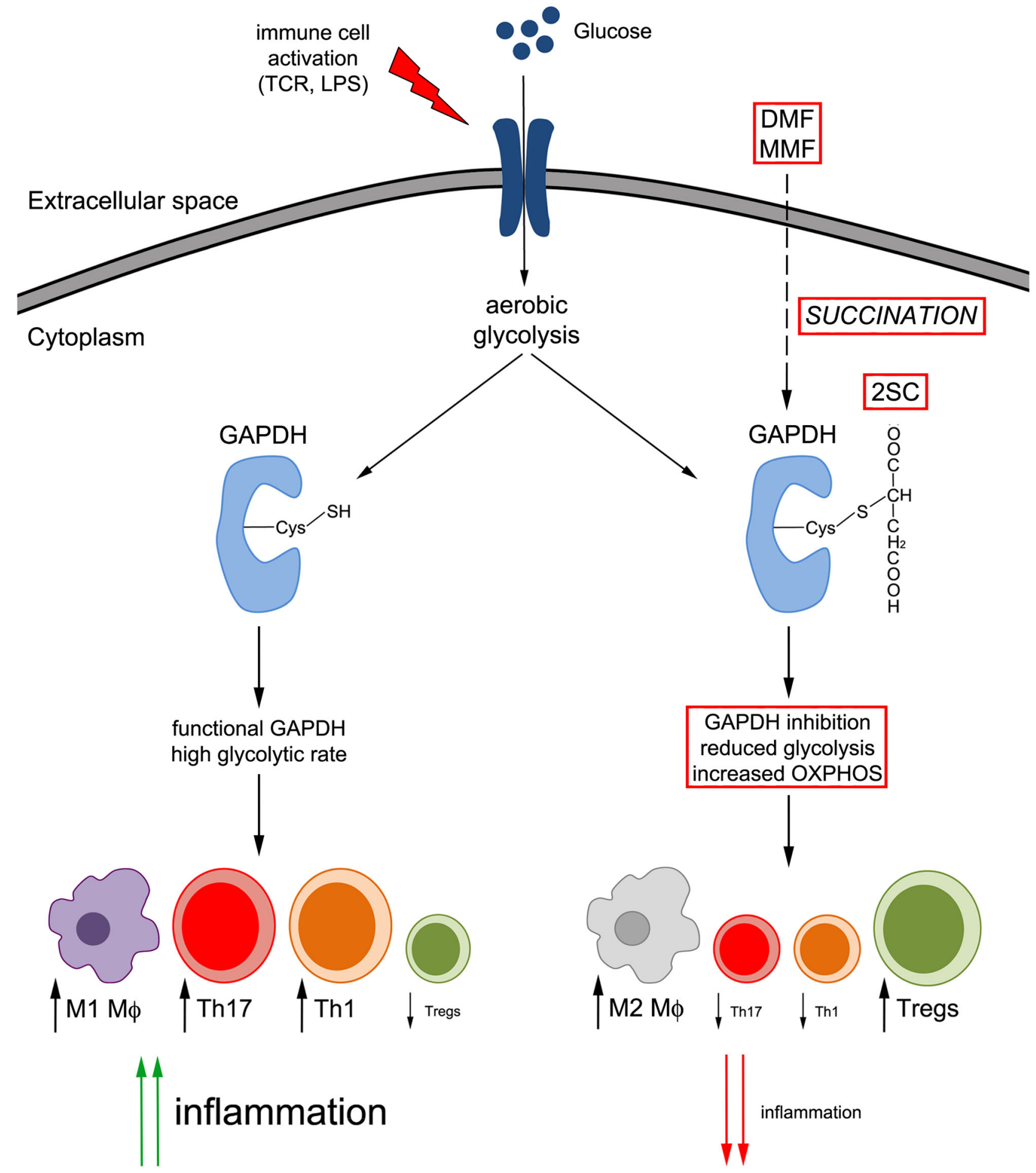

Fig. 1 GAPDH is a target for dimethyl fumarate. Following activation, innate and adaptive immune cells increase glucose uptake to fuel aerobic glycolysis, an essential step for their activation and effector functions, and GAPDH represents a rate-limiting enzyme in this pathway. Engagement of glycolysis favors the generation of pro-inflammatory immune cell subsets such as M1-polarized macrophages, Th1 and Th17 cells. DMF and MMF both alter the functionality of GAPDH, by inducing succination of Cys-152 in the enzyme active site. This modification inactivates GAPDH, causing a block in glycolysis, an increase in OXPHOS, the development of anti-inflammatory immune cell subsets such as Tregs and M2-polarized macrophages, and an overall inhibition of the inflammatory response. Abbreviations not present in the main text: TCR T-cell receptor, Cys cysteine, 2SC S-(2-succino)cysteine, M $\phi$ macrophage

demand. ${ }^{7}$ As expected, Kornberg and co-authors found that DMF, MMF and HA all inhibit Th1 and Th17 development while favoring Treg generation in vitro, confirming previous reports showing that inhibition of glycolysis impacts inflammation and that DMF alters
T cell subset in treated patients. ${ }^{1-3,7}$ Importantly, HA administration in mice inhibited the development of experimental autoimmune encephalomyelitis (EAE), a neuroinflammatory pathology that represents the main murine model of MS, confirming the 
potential of GAPDH and glycolysis inhibition to limit $T$ cellmediated inflammation in vivo.

This paper therefore sheds new light on the immunomodulatory activity of DMF and confirms that altering the glycolytic capacity of activated innate and adaptive immune cells, in this case via GAPDH inhibition, represents a valuable approach to modulate inflammatory responses in vivo. The paper also reinforces the concept that post-transcriptional protein modifications by intracellular or exogenous metabolites can impact immune responses by modulation of immune cell activation and function. ${ }^{9}$ Furthermore, autoimmune and inflammatory diseases may well be treatable using drugs that alter the metabolic state of immune cells, ${ }^{10}$ in this case by targeting glycolysis. This study therefore highlights the exciting prospect that targeting immunometabolism might lead to new treatments for a wide range of inflammatory diseases.

\section{REFERENCES}

1. Brück, J. et al. Exp. Dermatol. https://doi.org/10.1111/exd.13548 (2018).

2. Mills, E. A., Ogrodnik, M. A., Plave, A. \& Mao-Draayer, Y. Front. Neurol. 9, 5 (2018)

3. Kornberg, M. D. et al. Science 360, 449-453 (2018).

4. Merkley, E. D. et al. Mass. Spectrom. Rev. 33, 98-109 (2014).

5. Alderson, N. L. et al. Arch. Biochem. Biophys. 450, 1-8 (2006).

6. Piroli, G. G. et al. Biochem. J. 462, 231-245 (2014).

7. O'Neill, L. A., Kishton, R. J. \& Rathmell, J. Nat. Rev. Immunol. 16, 553-565 (2016).

8. Diebold, M. et al. J. Autoimmun. 86, 39-50 (2018).

9. Mills, E. L. et al. Nature 556, 113-117 (2018)

10. Bettencourt, I. A. \& Powell, J. D. J. Immunol. 198, 999-1005 (2017). 